\title{
Cambios en la representación social de la educación física en la formación inicial del profesorado Changes in the social representation of physical education during the initial teacher training \\ Alexandra Valencia-Peris, Jorge Lizandra \\ Universidad de Valencia(España)
}

\begin{abstract}
Resumen. En este trabajo se utiliza el relato autobiográfico para conocer la relación entre la Educación Física (EF) y cada uno de los estudiantes de un grupo de segundo curso del grado de magisterio en educación primaria. Concretamente se pidió que redactaran dos relatos, uno antes y otro después de haber cursado la asignatura troncal de didáctica de la EF en educación primaria, con el fin de conocer si se producían cambios en la concepción de la EF escolar tras haberla cursado. En el primer relato abundan recuerdos relacionados con el enfoque técnico y tradicional de la asignatura así como con la analogía EF-tiempo libre. Asimismo, destaca el papel del profesorado, los cambios en función de la etapa educativa y la discriminación en algunos grupos de alumnos. En el segundo relato aflora positivamente el hecho de haber aprendido y comprendido el valor educativo que posee la EF escolar, lo que ha supuesto un cambio transformador en las representaciones sociales del alumnado respecto a esta asignatura. Se concluye que conviene seguir dando voz al alumnado para, a partir de sus vivencias, creencias y actitudes, poder conformar experiencias educativas significativas que provoquen un cambio de paradigma.
\end{abstract}

Palabras clave: educación física; relato autobiográfico; educación superior, imaginario colectivo.

Abstract. In this paper, the autobiographical story is used to know the relationship between Physical Education (PE) and each one of the students from a second grade group of the degree in primary education. Specifically, they were asked to write two stories, one before and another after they had taken the core subject of didactics of PE in Primary education, in order to know if there were changes in the conception of the school PE after having completed it. In the first story there are many memories related to the technical and traditional approach of the subject as well as to the PE-free time analogy. Teachers' role is also highlighted as well, changes in terms of the educational stage and the emergence of marginalized groups. In the second story, the fact of having learned and understood the educational value of school PE has positively emerged, which has meant a transformative change in the social representations of students regarding this subject. In conclusion, it is advisable to continue giving voice to the students to create, based on their experiences, beliefs and attitudes, meaningful educational experiences that led to a paradigm shift.

Keywords: physical education; autobiographic tale; higher education, collective imagery.

\section{Introducción}

La concepción y la actitud que muestra el alumnado de magisterio de educación primaria sobre la educación física (en adelanteEF) se ve en gran medida condicionada por la representación social que tienen de la misma. Se entiende por representación social al conjunto de procesos cognitivos y sociales a través de los cuales los individuos estructuran un determinado concepto y lo convierten en sentido común, integrándolo como habitual en la realidad social colectiva (Moscovici, 1979). En relación con la EF, la representación social sería el conjunto de concepciones y creencias colectivas que emergen a partir de las interacciones desarrolladas, tanto en la asignatura como en el entorno próximo de la misma, principalmente la escuela y la comunidad. Las representaciones sociales en la clase de EF se configuran principalmente por el conjunto de vivencias que los estudiantes han experimentado en la asignatura durante el periodo de escolarización obligatoria (De la Torre, 2011). De ahí que la misma asignatura pueda ser muy diferente en función de variables como el momento vital o los distintos enfoques metodológicos otorgados por las profesoras o profesores que la hayan impartido. Del mismo modo, fuera de la escuela opera la influencia de un paradigma social y cultural en el que la EF ha estado tradicionalmente muy vinculada tanto a aspectos meramente motrices y corporales como a un discurso hegemónico basado principalmente en el rendimiento y la competición.

Por este motivo, desde la asignatura de didáctica de la educación física en educación primaria pretendemos mostrar una versión educativa de la asignatura escolar en sentido amplio, una EF preocupada por las experiencias motrices pero también por la integración detodoel alumnado, que facilite relaciones interpersonales desde el respeto a la diferencia.

La asignatura de EF, suele ser una asignatura que agrada a la mayoría de los estudiantes (Moreno \& Hellín, 2007). El hecho de que se lleve a cabo generalmente al aire libre, o al menos en un espacio distinto al aula y que sea una asignatura eminentemente práctica, son elementos que hacen que la EF cuente a priori con el beneplácito del alumnado.

Fecha recepción: 26-09-17. Fecha de aceptación: 04-02-18

Alexandra Valencia Peris

alexandra.valencia@uv.es
Diversos autores indican que aquellos adolescentes que tienen una percepción positiva y disfrutan de las clases de EF, son los que realizan más actividad física fuera del entorno escolar (Duncan, 1993; Mendoza, Sagrera \& Batista, 1994), logrando con ella satisfacer uno de los fines fundamentales de la EF (Portes \& Pacheco, 2010). No obstante, existen también evidencias de que esa percepción positiva hacia la asignatura no siempre perdura a lo largo de la escolarización o al menos no es unánimemente compartida por todas y todos los estudiantes. Por ejemplo, la EF suele agradar más a los chicos que a las chicas (Moreno, Rodríguez \& Gutiérrez, 2003), incluso parece ser que el interés decrece a medida que los estudiantes se van haciendo más mayores (Gutiérrez \& Pilsa, 2006).

Según la literatura son diversas las razones por las que puede surgir este desinterés, pero en la mayoría de los estudios, las principales causas se relacionan con el papel del profesor o la profesora en la asignatura. En unos casos se alude a la falta de variedad de contenidos ofrecidos (Sebire et al., 2013) o a la desatención de áreas como las expresivas o artísticas (Amado, Del Villar, Leo, Sánchez-Oliva, SánchezMiguel \& García-Calvo, 2014). En otros casos son algunos colectivos como las personas con menor habilidad o las chicas, las que perciben una sensación de abandono o rechazo, sentimientos que no hacen más que acrecentar los estereotipos de cultura del rendimiento o de género que habitualmente se le atribuyen a la EF (Blández, Fernández \& Sierra, 2007; Ruiz, Gutiérrez, Graupera, Linaza \& Navarro, 2001).

Tradicionalmente la asignatura de EF se ha centrado únicamente en la dimensión corporal, pasando por alto otras dimensiones esenciales que le otorguen una concepción educativa en sentido amplio (Devis \& Peiró, 2011). Sin embargo, los contenidos están inevitablemente vinculados a las relaciones interpersonales que sucedan en el contexto, por lo que sólo cuando se tiene la capacidad de ofrecer estrategias metodológicas que influyan positivamente en el contexto en el que se desarrollan, se podrá hablar de un valor educativo en sentido amplio. Al parecer, a pesar que en los últimos años se está produciendo un viraje de las propuestas de la EF hacia opciones más diversas, el peso de la tradición y de la percepción hegemónica de la asignatura sigue imponiéndose, aspecto que a menudo crea un sentimiento de crisis de identidad en la asignatura (Moreno-Doña, Valencia-Peris \& Rivera, 2016). En ese sentido conviene tener presente que la asignatura de $\mathrm{EF}$, independientemente de la amplitud conceptual en la que se desarrolle, debe mostrar una intención pedagógica a la que pueda atribuírsele un valor educativo. 
Para ello, debe ir siempre precedida de una justificación de sus contenidos que sea a su vez moralmente aceptable (Arnold, 1991). De ahí que el desempeño del profesorado en el proceso de enseñanza-aprendizaje se considere esencial, ya que influye directamente en la percepción del alumnado hacia la asignatura, teniendo esto un gran peso en su decisión de participar en actividades físico-deportivas en su tiempo libre (Torre, Cárdenas \& García, 2001). Podría decirse por tanto que las percepciones se aprenden en cierta medida y que encuentran su fundamento en las experiencias vividas, en este caso en las clases de EF (Moreno \& Hellín, 2002). Según Gutiérrez (2014), el recuerdo de malas experiencias en la EF escolar a menudo se relaciona con la falta de satisfacción hacia las actividades fisico-deportivas. En la misma línea, Beltrán, Devís, Peiró y Brown (2012) destacan las experiencias negativas como uno de los elementos que han dificultado su intención de realizar actividad física en el futuro.

Una manera de conocer las concepciones, los sentimientos y las predisposiciones hacia la EF es acudir a los relatos o historias de las y los estudiantes, puesto que las personas somos seres eminentemente narrativos, siendo por tanto la narración la manera más natural de contar y comprender las experiencias (Frank, 1995). A menudo las historias ayudan a organizar y encajar las experiencias y las situaciones vividas. Además, la información de los relatos no sólo resulta útil en el contexto en el que se desarrolla, sino que puede ser incluso transferible a otros contextos similares, dado que aunque las historias son individuales, el contenido arroja información del contexto social y cultural en el que suceden, por lo que las narraciones individuales son al mismo tiempo personales y sociales (Murray, 1999). De este modo, los relatos autobiográficos representan un compendio de aquello que el alumnado conoce o piensa acerca de la EF, así como de sus percepciones y de la intencionalidad de acción futura hacia la asignatura (De la Torre, 2011).

Consideramos fundamental comprender la visión de la EF y los significados sociales que han construido los y las estudiantes de magisterio durante su trayectoria formativa en la etapa escolar a partir de sus experiencias vividas. De este modo, el análisis reflexivo sobre las mismas puede convertirse en un eslabón estructurante en cualquier proceso formativo profesional docente, puesto que nos otorga la posibilidad de revelar los enfoques teórico-analíticos presentes en la disciplina y las representaciones sociales que subyacen en el pensamiento de los futuros docentes, con las respectivas implicaciones en los procesos de (re)producción pedagógica que quedarán plasmados en el momento que les toque desempeñar su labor docente (González, 2014).

Por tanto, el objetivo principal del presente trabajo es analizar mediante relatos elaborados por el alumnado cuáles son las representaciones sociales sobre la EF que tiene el alumnado que llega a Magisterio por un lado y, por otro, comprobar si tras cursar una asignatura específica de Didáctica de la EF existe a nivel global un cambio en las mismas.

\section{Contextualización de la experiencia}

La experiencia que presentamos tuvo lugar en el Grado en Maestro/ a en Educación Primaria de la Universitat de València, concretamente en un grupo de la asignatura obligatoria y cuatrimestral (6 créditos ECTS) Didáctica de la Educación Física en Educación Primaria de $2^{\circ}$ curso (antes de elegir especialidad o mención en el grado). El desarrollo de la misma comprendió el primer cuatrimestre del curso 2016/17 dónde había matriculados un total de 49 estudiantes (37 chicas y 12 chicos de edades comprendidas entre los 19 y los 21 años).

La materia universitaria está meditada y planteada para lograr que los estudiantes adquieran los conocimientos y las capacidades suficientes para proponer y dinamizar clases de EF en educación primaria, partiendo de una filosofía de trabajo que favorezca la integración y las relaciones interpersonales y que está basada en valores fundamentales como el respeto y la aceptación de la diferencia, así como la cooperación, el sentimiento de pertenencia al grupo y la solidaridad entre iguales. Además, se atiende a la adquisición de capacidades vinculadas a la acción motriz, así como a elementos de la cultura física, tratando de incorporar una visión crítica que favorezca la reflexión para que sean capaces de construir su propia opinión del significado de la EF. Con este planteamiento se pretende, en última instancia, aportar un equilibrio saludable entre las dimensiones físicas, psíquicas y sociales, desde un paradigma de justicia social, que finalmente repercutan en el buen desarrollo de las niñas y niños que vayan a tener a nuestros estudiantes como docentes en un futuro próximo.

Por lo que respecta a los contenidos que se trabajan durante la asignatura, conviene que estén alineados con la filosofía anteriormente descrita. En ese sentido, se da comienzo a la asignatura tratando de mostrar el valor educativo de la EF, para posteriormente tratar de comprender los distintos modelos de EF y en qué medida se alinean con las premisas fundamentales del modelo educativo con el quenos identificamos. Para este cometido, el conocimiento de la evolución histórica de la EF es de gran utilidad. También se estudia y analiza el currículum de la EF en educación primaria, de manera reflexiva y crítica, para ver las bondades y puntos de mejora del mismo. Finalmente se trabaja de manera teórico-práctica tanto los distintos elementos de una programación, como la propia metodología didáctica. Así, se ponen en práctica distintos modelos de enseñanza, para posteriormente centrarnos en aquellos modelos alternativos a las concepciones más tradicionales, porque entendemos que optimizan su valor educativo(Molina \& Beltrán, 2007). Concretamente se propone una EF inclusiva, orientada a una concepción amplia y dinámica de la salud, en la que se plantean lógicas de juego alternativas al modelo de competición, como la lógica cooperativa o paradójica. Todo tratando de favorecer la participación de todas las personas y reforzando el auto-concepto, la autonomía y las relaciones sociales.

Respecto al sistema de evaluación, se hace uso principalmente de una metodología de evaluación formativa y continua, en la que se obtienen tanto valoraciones como calificaciones durante todo el proceso de aprendizaje. Además, en cuanto a la calificación, los elementos prácticos y vivenciales tienen un equivalente al de elementos más teóricos. Concretamente, el 50\% de la calificación final se obtiene por la participación activa en clase (20\%) y el desarrollo de una unidad didáctica tutorizada en grupo (30\%), mientras que el otro $50 \%$ se consigue en una prueba sobre conocimientos teóricos (30\%) junto con la redacción de los dos relatos (20\%).

\section{Material y métodos}

Este trabajo se enmarca dentro de una investigación educativa, es decir, buscamos actuar directamente sobre la docencia con la intención de mejorarla (Blández, 2010). La investigación educativa indaga desde una perspectiva interna, es decir, parte de la propia práctica docente, considerando tanto al profesorado como al alumnado como participantes activos del proceso (Blández, 2000). Se diferencia así de la investigación sobre educación, donde se adopta una perspectiva externa donde la persona que investiga parte de una interpretación de la realidad y considera a profesorado y a alumnado como objeto de estudio.

\section{Recogida de datos e informantes}

Al principio de la asignatura se encomendó a los y las estudiantes una tarea en la que habían de escribir un breve relato autobiográfico (máximo 2 páginas) titulado «La Educación Física y yo» y remitirlo por correo electrónico al profesor. En el mismo relato, el cual tenía un carácter totalmente abierto, debían de señalar las percepciones, los significados y los recuerdos sobre lo que habían experimentado en la asignatura de EF en etapas escolares previas a la universitaria. La última semana de clases, tras haber completado la asignatura, se volvió a solicitar al estudiantado una reflexión en forma de relato y bajo el mismo título, en la que dieran cuenta de la percepción y el significado que tenía para ellos la EF escolar a partir de ese momento, es decir, tras haber cursado la materia universitaria. Se valoró tanto la relevancia del contenido de los relatos como la calidad y la corrección de la composición.

Los 48 estudiantes matriculados en la asignatura participaron en la experiencia y únicamente un alumno no entregó el segundo relato por lo que el porcentaje de participación fue del $99.1 \%$. Todos los participantes fueron informados de la posible utilización de sus relatos para una 
investigación educativa sobre la materia en la que estaban matriculados. Asimismo, se garantizó la utilización ética de los datos obtenidos y la privacidad y confidencialidad de las personas participantes. Por ello se han utilizado pseudónimos con el fin de preservar el anonimato de los y las participantes en la experiencia que se investiga.

\section{Procedimiento de análisis}

La aproximación metodológica cualitativa adoptada en este estudio y la naturaleza textual de los datos obtenidos condicionan la manera de efectuar su análisis, que implica un tratamiento que preserve la naturaleza textual de los mismos. El análisis cualitativo supone el examen sistemático del conjunto de elementos informativos con el objetivo de delimitar sus partes y establecer relaciones entre las mismas y el todo.

La primera tarea del análisis consistió en reducir los datos mediante la simplificación, resumen y selección de la información una vez leídos todos los relatos. Además, el análisis se ajustó a los procedimientos de fragmentación y articulación de la «Grounded Theory» (Glasser \& Strauss, 1967; Strauss \& Corbin, 2002; Valles, 2007), incluyendo espirales de profundización en las que se iba retrocediendo y avanzando de forma continua a lo largo del proceso hasta construir una teoría lo más coherente posible.

Como señalan Strauss y Corbin (2002, p. 110), la primera codificación de los datos consistió en «el proceso analítico, por medio del cual se identifican los conceptos y se descubren en los datos sus propiedades y dimensiones». El análisis consistió en identificar unidades textuales de los comentarios de los y las estudiantes y dotarlas de sentido conjunto agrupándolas y clasificándolas en unidades temáticas, a través de un proceso de categorización inductivo, tal y como se indica en la literatura especializada (Huberman \& Miles, 1994; Rodríguez, Gil \& García, 1996). Para la categorización seguimos un proceso de inducción analítica que, como señalan Goetz y LeCompte (1988), consiste en la extracción de las categorías del conjunto total de datos. De esta manera, las categorías de nuestro análisis emergieron de la información obtenida en los trabajos del estudiantado, es decir, no se partió de un esquema previo de categorías para el estudio, sino que estas surgieron del examen de la información procedente de los ensayos entregados. Concretamente, se trata de categorías que a posteriori son producto de la reducción de datos textuales. La Figura 1 muestra las categorías que emergieron tras el análisis inductivo de los relatos iniciales y los relatos finales.

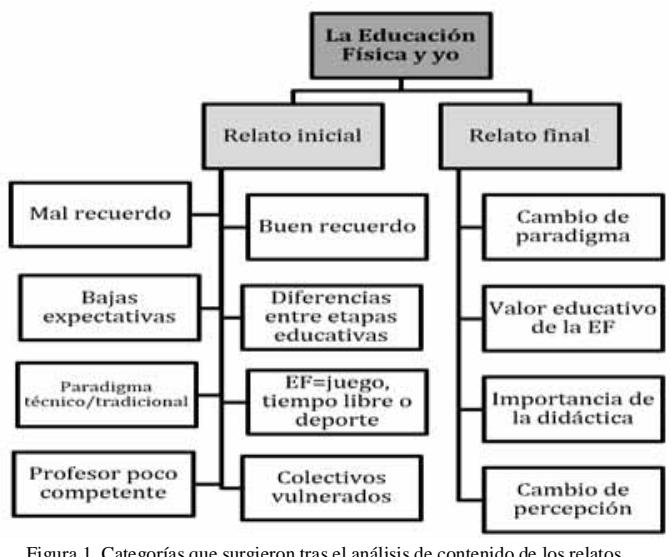

\section{Resultados y discusión}

Este apartado lo estructuramos en dos secciones. La primera de ellas dedicada a presentar las percepciones, significados y recuerdos que emergieron de los relatos autobiográficos antes de iniciar la materia universitaria de estudio. El segundo profundiza en los cambios experimentados en el alumnado en relación a lo que significa para ellos la EF escolar tras cursar la materia de Didáctica de la EF en Educación Primaria.
¿Cuáles son las experiencias del alumnado en EF antes de entrar al Grado de Magisterio?

Empezando por las categorías que emergieron en el análisis de los relatos autobiográficos que se solicitaron al inicio de la asignatura son varios los aspectos en los que coinciden la mayoría de estudiantes. Muchos de ellos mantienen un buen recuerdo de la asignatura de EF al referirse a ella como «aquella que se salía de la rutina, didáctica, activa, donde te divertías haciendo deporte (Pau)» o que «siempre ha sido y será una de las asignaturas escogida por los niños y niñas como la más divertida y la que más gusta (Miriam)». En este sentido, trabajos como el de Moreno y Hellín (2007) han demostrado que la EF es una de las asignaturas que más gusta al alumnado. No obstante, encontramos numerosas referencias que otorgan a la EF un mal recuerdo-«aquella en la que pensabas y te entraba dolor de cabeza (Javier)», incluso éste les hace mantener un miedo que se plasma en la inseguridad que le provocaba tener que cursar de nuevo esta asignatura en el grado de Magisterio. Según apuntan Monforte y Pérez-Samaniego (2017), en un estudio etnográfico sobre el miedo y la EF, éste puede marcar la manera de vivir, aprender y crecer en esta materia, por lo que podría incluso condicionar la experiencia de los alumnos y alumnas en la asignatura de Didáctica de la Educación Física. De hecho, estas vivencias negativas implican que parte del alumnado muestre bajas expectativas de cara a cursar la asignatura que presentamos como objeto de investigación: «Esa mala experiencia hace que no tenga buenas expectativas con la asignatura (Clara)».

Al igual que lo apuntado por Gutiérrez y Pilsa (2006) en relación al decreciente agrado y disfrute por la EF conforme aumenta la edad del alumnado, la gran mayoría de participantes de este estudio hace especial hincapié en las enormes diferencias que han encontrado y experimentado al pasar de una etapa educativa a otra. Concretamente, desde Educación Infantil hasta Bachillerato pasando por Educación Primaria y Educación Secundaria Obligatoria. Estas diferencias radican principalmente en el tipo de contenidos y metodologías con los que se han ido encontrando y que, normalmente, han evolucionado a peor desde una EF basada en el juego (la mayoría de veces libre o dirigido) a una EF fundamentada en las pruebas físicas o aprendizaje de diversos deportes (Sánchez Bañuelos, 1984) junto con la inclusión en estas últimas etapas de exámenes teóricos. Este último aspecto es mal visto por los participantes por no encontrar la utilidad de estudiar los diferentes reglamentos deportivos que es en lo que principalmente se basaban las pruebas teóricas. Algunas citas que fundamentan este hallazgo son:

«En la etapa de infantil, las actividades que realizaba eran principalmente de carácter lúdico, pues en clase los maestros nos hacían bailar, cantar e incluso preparábamos varias obras teatrales para las fiestas de Navidad (Joan)».

«Al llegar a la primaria fue cuando la educación física degeneróun poco (Antonio)».

«Una vez empecé la ESO las clases eran más técnicas [...], no hacíamos juegos generales como en Primaria, sino que hacíamos actividades específicas para trabajar lo que tocaba en cada sesión (Silvia)».

En este sentido, la mayoría de experiencias se sitúan bajo el paraguas del enfoque técnico o tradicional de la EF, donde la enseñanza se basa en actividades fácilmente observables y medibles para establecer los objetivos conductuales (Devís \& Molina, 2001). En este sentido, los contenidos tradicionales del campo, ligados al rendimiento físico y deportivo (Molina \& Beltrán, 2007), se ajustan perfectamente a esta racionalidad técnica, especialmente la condición física y las habilidades específicas de los deportes:

«El calentamiento siempre consistía en dar vueltas a la cancha y después nos dedicábamos al deporte concreto que tocara ese día. Pasaron de ser juegos divertidos a la especialización de un deporte siguiendo las normas (Inma)».

Asimismo, encontramos muchos testimonios donde se observa una evaluación objetiva basada en la condición física del alumnado o en sunivel de habilidad motriz que se transformaba directamente en calificación (Hernández Álvarez \& Velázquez, 2005). Esta percepción es 
compartida por otros estudios (De la Torre, 2011) en los que incluso se indica que, fruto del enfoque de evaluación que han experimentado durante su escolarización, el estudiantado de formación inicial del profesorado no es capaz de distinguir claramente entre la evaluación y la calificación; a pesar del cambio de enfoque que se viene implementando en la evaluación de la EF hacia sistemas más democráticos y basados en el proceso de aprendizaje (López Pastor et al., 2006):

«A pesar que siempre me ha gustado el deporte, nunca me he llevado especialmente bien con la asignatura de EF. Para mí, siempre fue una dificultad cumplir con los requisitos físicos que se exigían(Martí)».

En estas vivencias ha predominado una racionalidad tecnocrática (Fernández-Balboa \& Muros, 2006), es decir, un espacio donde se priorizan los hechos objetivos, los cálculos y las medidas antes que los valores éticos y los sentimientos humanos. De esta forma, el elitismo, la competición y el individualismo han sido elementos que se han transmitido al alumnado participante mediante currículum oculto (Bain, 1985; Jackson, 1968), reproduciendo así conductas discriminatorias por motivos de nivel de habilidad motriz. No obstante, también hay algunos estudiantes que señalan haber disfrutado aprendiendo contenidos relacionados con la expresión corporal, los juegos cooperativos o los deportes alternativos, aspecto que viene a demostrar la yuxtaposición de corrientes o enfoques existentes en la EF escolar (Hernández Álvarez, 1996):

«Alberto (pseudónimo) fue el profesor que me hizo ver la educación física de forma diferente. Esto es debido a que sus clases eran más originales, más divertidas, con muchos más juegos de cooperación y donde desarrollaba otras capacidades como el equilibrio, la coordinación o el compañerismo (Antonio)».

Por otro lado, son también muchos los alumnos que han vivido la EF como equivalente a «tiempo libre» lo cual gusta por el protagonismo que pueden alcanzar en un momento determinado pero también contribuye a disminuir el estatus que se otorga al compararlo con otras asignaturas con una programación y metodologías mucho más cerradas (Carr, 1983; Sáenz-López, 1999): «En primaria [... .] esperaba con ganas que tocase esta asignatura porque era la única que nos dejaba libertad para realizar lo que quisiéramos (Inma)».

Por último, han surgido dos categorías en los relatos iniciales que están relacionadas y que pueden ser determinantes a la hora de configurar un mal recuerdo de la EF. La primera es haber coincidido con un profesor o profesora de EF poco competente o con carencias profesionales:

«Tuve la mala suerte de tener un profesor que parecía un «general» era muy arbitrario con nosotros y súper exigente sin tener en cuenta el esfuerzo personal (Javier)».

«Los ejercicios del examen se realizaban delante de toda la clase, lo que aportaba gran cantidad de nervios y vergüenza, más aún cuando expresaba la nota en público y articulaba comentarios desafortunados (Inma)».

Y es que, tal y como apunta Hernández Álvarez (1996), el profesorado de EF ha significado un elemento determinante en la construcción histórica y social de la asignatura. Sus vivencias escolares en esta área, la formación inicial recibida, sus expectativas profesionales y los valores que le confieren al cuerpo y a la actividad física, en el contexto educativo, se configuran como elementos fundamentales que se interrelacionan y desde los que se elaboran juicios de valor, se construyen y se desarrollan concepciones, teorías y creencias personales que posteriormente mantienen y transmiten en sus acciones docentes.

En segundo lugar, haberse sentido excluido o discriminado por motivos de género, discapacidad, sobrepeso o baja condición física, que es a lo que hemos denominado colectivos vulnerados(Lentillon, Cogérino \& Kaestner, 2006; Núñez, Campos, Alfaro \& Holst, 2013) también ha contribuido en la conformación de un mal recuerdo de la EF escolar. Estos colectivos pueden sentirse discriminados de forma directa o indirecta (Moreno-Doña et al., 2016). De manera directa cuando son los propios maestros quienes, mediante sus actuaciones, excluyen por motivos de género (ej: «Siempre se escogía (primero) a los chicos y a las chicas más deportistas [...] y por ello, quizás, algunos de los alumnos se pueden sentir excluidos (Miriam)»). Y de manera indirecta al hacer caso omiso del alumnado que no participa o manifiesta necesidades educativas especiales (ej: Muchas veces no se tomaba en serio mis dolores de espalda (crónicos), añadiendo siempre que me dolía la espalda porque estaba creciendo, y que me podía esforzar más (Silvia)»).

En general, los recuerdos del alumnado participante representan una mezcla de sentimientos en relación con la EF. En general se trata de una asignatura que gusta por su propia naturaleza y contexto en el que se realiza, pero al mismo tiempo, por el tipo de metodologías y contenidos y cómo éstos se han llevado a la práctica, ha generado un enfoque tibio o poco gratificante, sobre todo en aquellas personas que no se caracterizan por ser el alumno o alumna más hábil motrizmente.

¿Cuáles son las experiencias del alumnado en EF tras cursar la asignatura de Didáctica de la Educación Física?

Tras su paso por la asignatura de Didáctica de la EF en Educación Primaria el alumnado afirma haber comprendido mejor el papel que juega la EF en el actual sistema educativo pasando de una concepción técnica a una práctica (racionalista) e incluso crítica o transformadora de la materia (Devís \& Molina, 2001).

Los comentarios hacen alusión, en gran parte, al valor educativo e intrínseco propio de la EF (Arnold, 1991) y que anteriormente desconocían: «la EF es una asignatura muy completa y que para mí trabaja con un plano integral de los seres humanos, desde los aspectos físicos, pasando por multitud de áreas hasta la moral humana (Laura)». En este sentido, comprenden mejor la justificación de su presencia en el currículum siempre y cuando ésta sea sensible a la integración de todo el alumnado. Así, y según sus percepciones en la asignatura, han de tener cabida todos y todas las estudiantes indistintamente de sus características, dificultades y ritmos de aprendizaje, partiendo de la premisa de que cualquier persona es educable en un entorno ordinario, respondiendo a todo el alumnado sin discriminación de ningún tipo (Ríos, 2009). Para este cometido, es necesario formar al futuro maestro de EF para que tenga en cuenta diferentes estrategias de atención a la diversidad, que pueda utilizar una metodología acorde a cada tipo de actividad, que esté en condiciones de seleccionar aquéllos contenidos que marca el currículum y que consideren más relevantes en cada situación, que sepan llevar a cabo una evaluación formativa y compartida adaptada a las características del alumnado, y que puedan apoyarse siempre en una educación basada en valores (cooperación, respeto, fair-play, empatía...). Tras experimentar estos procesos formativos, el bajo estatus que inicialmente otorgaban muchos participantes a la EF ha aumentado considerablemente: «me alegra que una asignatura como ésta, que en la mayoría de ocasiones ha estado (y todavía sigue estando) «infravalorada», sea una de las pocas que nos haya hecho plantearnos de manera crítica ciertos aspectos con los que tendremos que lidiar [...] en un futuro no muy lejano (Inma)».

El alumnado coincide en señalar la importancia que tiene estudiar la didáctica para comprender mejor la EF y llevar a cabo una enseñanza de calidad (Moreno-Doña et al., 2016): «Nunca me había planteado la cantidad de cosas que hay que tener en cuenta un maestro para dar clase (atención a la diversidad, interdisciplinariedad, transversalidad, metodología... ) (Joan)». La mayoría resalta como aspecto muy positivo la oportunidad brindada en la asignatura en la que, por grupos, han tenido que diseñar una unidad didáctica y desarrollar de forma práctica una sesión en el Aula de Educación Física con el resto de sus compañeros: «al llevar a cabo la realización de una unidad didáctica en la que tratamos un bloque de contenidos de la asignatura de EF para la Educación Primaria, he aprendido lo importante que es tener en cuenta cada una de las diferencias que hay entre los niños y niñas que podemos encontrar en las aulas (alumna)». Esta tarea ha permitido al alumnado ser protagonista de su aprendizaje de una forma aplicada e involucrarse desde el principio hasta el final bajo la orientación del tutor, lo que entraría dentro del sistema de evaluación formativa (López-Pastor et al., 2006) propuesto en la asignatura.

También se acentúa positivamente el hecho de haber dedicado una sesión en exclusiva a tratar el miedo en la EF (tras observar que muchos 
participantes mencionaban esta emoción negativa en sus relatos iniciales): «me gustó mucho que en una clase se tratara el tema del miedo a la EF, realmente me sentí muy identificada porque yo he sentido lo que es pasar malos momentos cuando venía la evaluación práctica de la asignatura (Lucía)». En este sentido, pensamos haber contribuido a ampliar el repertorio de historias que según Monforte y Pérez-Samaniego (2017) permiten al profesorado reconocer el miedo como un componente de sus prácticas pedagógicas, lo que puede ayudar a generar una comprensión más profunda y sensible de la acción docente, tal y como ha ocurrido en la asignatura.

Aunque existen alumnos cuyos relatos revelan que su paso por la asignatura no ha supuesto un cambio en su percepción, posiblemente porque no mantenían malos recuerdos sobre la EF, nos gustaría destacar en último lugar algunos comentarios de estudiantes que a nuestro parecer reflejan ese cambio en la percepción de la asignatura y que se deben al efecto transformador que ha supuesto cursar la materia universitaria:

«Tengo que admitir que mi opinión ha cambiado más de lo que me esperaba. Me gustaría que las personas que piensan como yo, cursaran esta asignatura (Ana)»

«Puedo decir que esta asignatura ha superado, y con creces, mis expectativas. Tengo que confesar que me planteo coger la especialidad de Educación Física, a pesar de que tenía pensado hacer otra especialidad(Núria)».

Como limitaciones de esta investigación cabe apuntar que se podrían haber tenido en cuenta las diferentes percepciones, así como los efectos en el cambio, según las identidades de género del alumnado (Devís-Devís, Fuentes \& Sparkes, 2005). Asimismo, haber realizado un análisis que no ha tenido en cuenta los cambios individuales, sino a nivel global, puede hacer que se pierdan datos relevantes entre el antes y el después de la relación de cada uno de los y las estudiantes con la EF. También hubiera sido interesante lograr una triangulación de datos recogiendo mayor información a través de entrevistas en profundidad a participantes de interés, mediante observación participante o grupos de discusión.

\section{Conclusiones}

Coincidimos con González (2014) en que si sostenemos que la educación es un bien social que tiene la posibilidad de dar paso a una sociedad más justa, inclusiva y más feliz, han de provocarse profundas transformaciones educativas en la formación inicial de los docentes además de cambios estructurales a nivel de país, de tal modo que aseguremos una educación de calidad. En los Grados de Magisterio concretamente debería impulsarse la innovación en los planes formativos junto con una formación que radique en el análisis crítico de las propias representaciones que han construido los futuros docentes acerca del quehacer disciplinar y las funciones que cumple la EF, en este caso, en la escuela. En esta línea y tras llevar a cabo esta investigación podemos decir que las percepciones del alumnado en relación con la EF han cambiado sustancialmente tras su paso por la asignatura de Didáctica de la Educación Física. Nos hemos encontrado con un alumnado diverso que, aun coincidiendo en que el enfoque de las clases de EF era predominantemente técnico y orientado a contenidos excesivamente deportivizados (De la Torre, 2011), en algunos casos manifestaba sentir buenos recuerdos respecto a esta materia pero también otros muchos que han tenido vivencias negativas. Podemos concluir, por tanto, que partir de las experiencias previas y recuerdos del alumnado para abordar una asignatura que se basa en la didáctica de la EF, asignatura que ha evolucionado tanto en tan poco tiempo, ha supuesto un punto de partida sobre el que enfocar la asignatura logrando un cambio de mentalidad en el imaginario colectivo o representación social de lo que significa hoy en día la EF.

\section{Referencias}

Amado, D., Del Villar, F., Leo, F. M., Sánchez-Oliva, D., SánchezMiguel, P.A., \& García-Calvo, T. (2014). Effect of a Multi-Dimen- sional Intervention Programme on the Motivation of Physical Education Students. Plos One, 9(1): e85275.

Arnold, P. J. (1991). Educación Física, movimiento y currículum. Madrid: Morata.

Bain, L. (1985) The Hidden Curriculum Re-examined. Quest 37, 14553.

Beltrán, V. J., Devís, J., Peiró, C., \& Brown, D. H. K. (2012). When physical activity participation promotes inactivity: Negative experiences of Spanish adolescents in physical education and sport. Youth \& Society, 44(1), 3-27.

Blández, J. (2000). La investigación-acción: un reto para el profesorado. Barcelona: Inde.

Blández, J. (2010). La clase de educación física: escenario de la investigación. En C. González y T. Lleixà (coords.), Educación Física. Investigación, innovación y buenas prácticas, pp. 43-58. Barcelona: Graó.

Blández, J; Fernández, E; \& Sierra, M. A. (2007). Estereotipos de género, actividad física y escuela: La perspectiva del alumnado. Profesorado. Revista de currículum y formación del profesorado, 11(1), 1-21.

Carr, D. (1983). On physical education and education significance. Momentum, 8(3), 21-24.

De la Torre, E. (2011). Significados de la Educación Física en el ámbito educativo. Un estudio desde las percepciones de los estudiantes de Magisterio. Universidad de Granada. Tesis doctoral inédita.

Devís-Devís, J., Fuentes, J., \& Sparkes, A. (2005). ¿Qué permanece oculto del currículum oculto? Las identidades de género y de sexualidad en la educación física. Revista iberoamericana de educación, 39, 73-90.

Devís, J., \& Molina, J.P. (2001). Los estudios del currículum y la Educación Física. En Vázquez, B. (coord.), Bases educativas de la actividad física y el deporte, pp. 243-276. Madrid: Síntesis.

Devís, J., \& Peiró, C. (2011). Sobre el valor educativo de los contenidos de la educación física. Tándem: didáctica de la educación física, 35, 68-74.

Duncan, S. (1993). The role of cognitive appraisal and friendship provisions in adolescents' affect and motivation toward activity in physical education. Research Quarterly for Exercise and Sport, 64(3), 314-323.

Fernández-Balboa, J. M., \& Muros, B. (2006). The hegemonic triumvirate-Ideologies, discourses, and habitus in sport and physical education: Implications and suggestions. Quest, 58(2), 197-221.

Frank, A. (1995). The wounded storyteller. Nuevo York: Sage.

Glasser, B., \& Strauss, A. (1967). The discovery of grounded theory: Strategies for qualitative research. Chicago: Aldine.

Goetz, J. P., \& Le Compte, M. D. (1988). Etnografía y diseño cualitativo en investigación educativa. Madrid: Morata.

González, L. (2014). Representaciones sociales de los y las estudiantes de pedagogía sobre la Educación Física escolar en Chile. Tesis Doctoral. Universidad de Granada.

Gutiérrez, M. (2014). Relaciones entre el clima motivacional, las experiencias en educación física y la motivación intrínseca de los alumnos. Retos. Nuevas tendencias en Educación Física, Deporte y Recreación, 26, 9-14.

Gutiérrez, M., \& Pilsa, C. (2006). Actitudes de los alumnos hacia la Educación Física y sus profesores. Revista Internacional de Medicina y Ciencias de la Actividad Física y del Deporte, 6(24), 212228.

Hernández Álvarez, J. L. (1996). La construcción histórica y social de la Educación Física: el currículo de la LOGSE, ¿una nueva definición de la Educación Física escolar? Revista de Educación, 311, 5176.

Hernández Álvarez, J. L. \& Velázquez, R. (2005). La educación física y su imagen social a la luz de las prácticas de evaluación y calificación del aprendizaje. Tándem: Didáctica de la Educación Física, 17, 720. 
Huberman, A. M., \& Miles, M. B. (1994). Data Management and Analysis Methods. En K. Denzin \& Y. S. Lincoln(Eds.), Handbook of qualitative research, pp. 428-444. California: Sage.

Jackson, P. (1968). La vida en las aulas. New York: Holt, Rinehart, and Winston.

Lentillon, V., Cogérino, G., \& Kaestner, M. (2006). Injustice in physical education: gender and the perception of deprivation in grades and teacher support. Social Psychology of Education, 9(3), 321-339.

López Pastor, V. M., Monjas Aguado, R., Gómez García, J., López Pastor, E. M., Martín Pinela, J. F., ... Martín, M. I. (2006). La evaluación en educación física. Revisión demodelos tradicionales y planteamiento de una alternativa. La evaluación formativa y compartida. Retos. Nuevas tendencias en educación física, deporte y recreación, 10, 31-40.

Mendoza, R., Sagrera, M. R., \& Batista, J. M.(1994). Conductas de los escolares españoles relacionadas con la salud (1986-1990). Madrid: CSIC.

Molina, J., \& Beltrán, V. (2007). Incompetencia motriz e ideología del rendimiento en educación física: el caso de un alumno con discapacidad intelectual. Motricidad: Revista de Ciencias de la Actividad Física y del Deporte, 19, 165-190.

Monforte, J., \& Pérez-Samaniego, V. (2017). El miedo en Educación Física: una historia reconocible. Movimento, 23(1), 85-100.

Moreno, J. A. \& Hellín, M. G. (2007). El interés del alumnado de Educación Secundaria Obligatoria hacia la Educación Física. Revista Electrónica de Investigación Educativa, 9(2), 1-20.

Moreno, J. A., \& Hellín, P. (2002). ¿Es importante la educación física? Su valoración según la edad del alumno y el tipo de centro. Revista Internacional de Medicina y Ciencias de la Actividad Física y del Deporte, 2(8), 298-319,

Moreno, J. A., Rodríguez, P. L. \& Gutiérrez, M. (2003). Intereses y actitudes hacia la Educación Física. Revista Española de Educación Física, $\operatorname{IX(2),14-28.}$

Moreno-Doña, A., Valencia-Peris, A., \& Rivera-García, E. (2016). La Educación Física Escolar en tres centros educativos de Chile: una caracterización de sus prácticas docentes. Qualitative Research in Education, 5(3), 255-275. doi:10.17583/qre.2016.2105
Moscovici, S. (1979). El psicoanálisis, su imagen y su público. Buenos Aires: Huemul.

Murray, M. (1999). The stories nature of health and illness. En M. Murray \& K. Chamberlain (Eds.), Qualitative health psychology, pp. 47 63. Londres: Sage.

Núñez, H., Campos, N., Alfaro, F., \& Holst, I. (2013). Las creencias de la docente de educación física sobre la obesidad en la niñez de edad escolar. Revista Electrónica Educare, 17(2), 5-30.

Portes, M., \& Pacheco, J. (2010). Percepción de los objetivos de la educación física en el currículum escolar desde la perspectiva de los estudiantes. Revista Horizonte Ciencias de la Actividad Física, 1(1), 44-53.

Ríos, M. (2009). La inclusión en el área de Educación Física en España. Análisis de las barreras para la participación y aprendizaje. Ágora para la Educación Física y el Deporte, 9, 83-114.

Rodríguez, G., Gil, J., \& García, E. (1996). Metodología de la investigación cualitativa. Málaga:Aljibe.

Ruiz, L. M., Gutiérrez, M., Graupera, J. L., Linaza, J. L., \& Navarro, F. (2001). Desarrollo, Comportamiento Motor y Deporte. Madrid: Síntesis.

Sáenz-López, P. (1999). La importancia de la Educación Física en Primaria. Apunts. Educación Física y Deportes, 57, 20-31.

Sánchez Bañuelos, F. (1984). Bases para una didáctica de la Educación Física y el Deporte. Madrid: Gymnos.

Sebire, S. J., Kesten, J. M., Edwards, M. J., May, T., Banfield, K., Tomkinson, K., ... Jago, R. (2016). Using Self-determination Theory to promote adolescent girls' physical activity: Exploring the theoretical fidelity of the Bristol Girls Dance Project. Psychology, Sport \&Exercise, 24, 100-110.

Strauss, A., \& Corbin, J. (2002). Bases de la investigación cualitativa: Técnicas y procedimientos para desarrollar la teoría fundamentada. Medellín: Universidad de Antioquia.

Torre, E., Cárdenas, D., \& García, E. (2001). Las percepciones que se derivan de las experiencias recibidas en las clases de educación física y su repercusión en los hábitos deportivos en el alumnado de bachillerato. European Journal of Human Movement, 7, 95-112.

Valles, M. (2007). Técnicas cualitativas de investigación social: Reflexión metodológica y práctica profesional. Madrid: Síntesis.

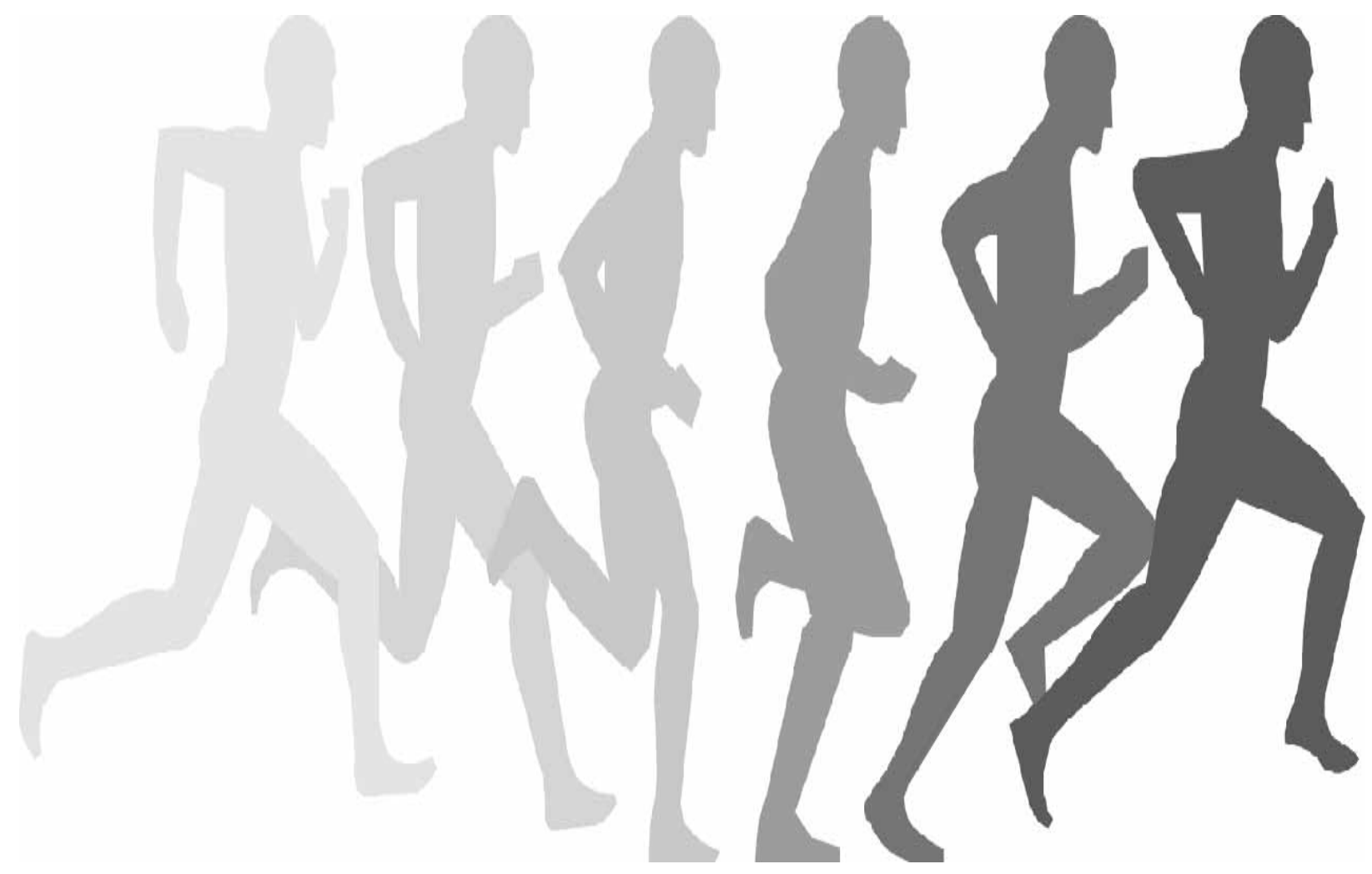

